\title{
Endomarketing: Satisfacción Laboral Desde La Perspectiva De Género Y Edad
}

\author{
Dra. Adriana Méndez Wong \\ Universidad Autónoma de Coahuila, Mexico \\ Mtro. Didier Varela Castro \\ Universidad del Valle de México
}

Doi:10.19044/esj.2018.v14n31p112 URL:http://dx.doi.org/10.19044/esj.2018.v14n31p112

\begin{abstract}
This paper focuses on analyzing the influence of the age and genre in the labor satisfaction of the sales professionals in the Southeastern region of Coahuila. The study makes use of a descriptive and quantitative approach. A questionnaire of 9 items was applied with Likert scale of 10 alternative answers. The study sample shows that 399 individuals were randomly selected. The findings of this study suggest that labor satisfaction is related to the genre, which shows that the samples of the men are more satisfied than the women. The recompensed salary is also a decisive factor on the feminine genre when they express their dissatisfaction with their job. In regards to the age, there are no statistically significant differences between those interviewed. In conclusion, the labor satisfaction is independent from the seller's age.
\end{abstract}

Keywords: Marketing, Endomarketing, Labor satisfaction

\section{Resumen}

El objetivo de este estudio es analizar la influencia que tienen la edad y el género en el nivel de satisfacción laboral de los profesionales de ventas en la Región Sureste de Coahuila. La investigación es de tipo descriptivo y cuantitativo, aplicándose un cuestionario de 9 ítems con escala de Likert de 10 alternativas de respuestas. La muestra es de 399 sujetos seleccionados aleatoriamente. Los hallazgos sugieren que la satisfacción laboral si está relacionada con el género, destacando que los hombres de la muestra están más satisfechos en comparación con las mujeres. El salario remunerado también es un factor decisivo para el género femenino al expresar su insatisfacción con el empleo. Respecto a la edad no se encuentran diferencias estadísticamente significativas entre los entrevistados, por lo que se concluye que la satisfacción laboral es independiente de la edad del vendedor. 
Palabras Claves: Mercadotecnia, Endomarketing, Satisfacción laboral

\section{Introducción}

El marketing debe formar parte de la filosofía de la empresa. La orientación y alineación de todas las actividades de la organización deberán estar centradas en la satisfacción de los clientes.

Un vendedor satisfecho con su trabajo, con su salario, con sus compañeros, con su jefe inmediato, con la empresa, con su ambiente de trabajo y sobre todo consigo mismo, tendrá un mejor desempeño que un compañero que no tenga estos satisfactores, ya que como dice un proverbio chino "un hombre que no sabe sonreír, no puede abrir una tienda" (tomado del libro de Dale Carnegie, "Cómomo hacer amigos y dominar a los demás" ( Carnegie, 1996)).De igual manera, al desarrollar una venta en condiciones positivas es muy posible que se haya ganadoun cliente de por vida.

\section{Revisión de literatura}

En la mercadotecnia tradicional, se toman en consideración factores tales como el cliente, el producto entre otros. En el énfasis del Endomarketing o Mercadotecnia Interna, el empleado es considerado como un cliente interno, teniendo necesidades, deseos y problemas; en tanto el trabajo es entendido como el producto que adquiere el cliente interno, el cual tendrá que satisfacer las necesidades y deseos de los empleados. Mientras que este último (cliente interno) esté satisfecho, mejor satisfará las necesidades y deseos del cliente externo (Varoglu \& Eser, 2006).

La mercadotecnia interna es el marketing realizado por una compañía de servicios para capacitar y motivar eficazmente a los empleados de contacto con los clientes y a todo el personal de servicios de apoyo para que trabajen en equipo con el fin de lograr la satisfacción del cliente (Kotler \& Armstrong, 2008).

La Mercadotecnia Interna requiere que todos los participantes de la empresa adopten los conceptos y objetivos de la mercadotecnia y participen en la selección, la generación y la comunicación de valor para el cliente (Kotler \& Keller, 2006).

Para Horacio Marchand (1998) para vender afuera, hay que vender adentro. Antes de posicionar afuera, hay que posicionar adentro. La venta empieza por casa; orgullo organizacional, gente comprometida, una clara visión, convencimiento, pasión, entrega.

Las empresas con un marketing sobresaliente procuran mantener satisfechos a sus clientes. Los clientes satisfechos vuelven a comprar, y comunican a otros sus experiencias positivas con el producto o servicio (Kotler \& Armstrong, 2008). 
La satisfacción de la clientela es la condición esencial para el establecimiento de una relación comercial perdurable. Si los usuarios están hasta tal punto satisfechos que por ello aumenta su deseo del producto, la consecuencia será un aumento del número de los clientes (afianzamiento de la fidelidad y captación en perjuicio de la competencia) y/o un crecimiento del ritmo de compra, de las cantidades consumidas, de la notoriedad, etc. (Serraf, 1988).

\section{Metodología}

Tabla 1. Método 1

\begin{tabular}{|c|c|}
\hline Tipo de investigación & Cuantitativa, descriptiva y transversal no experimental \\
\hline Tamaño de la Muestra & 399 sujetos \\
\hline Procedimiento de Muestreo & Aleatorio simple \\
\hline Error muestral & $4.0 \% ; \mathrm{p}=\mathrm{q}=0.5 ;$ nivel de confianza $95 \%$ \\
\hline Método de trabajo de campo & Encuesta auto administrada \\
\hline Cuestionario & Cuestionario estructurado de 9 ítems \\
Contexto & Las preguntas son categóricas y la escala de respuesta es \\
tipo Likert del 1 al 10 \\
Región Sureste de Coahuila, México
\end{tabular}

Fuente: Elaboración propia.

\section{Preguntas de investigación}

¿Influye el género en el grado de satisfacción laboral del vendedor?

¿Influye la edad en el grado de satisfacción laboral del vendedor?

\section{Hipótesis}

Ho: La satisfacción laboral del empleado con la empresa no está relacionada con el sexo del vendedor.

H1: La satisfacción laboral del empleado con la empresa sí está relacionada con el sexo del vendedor.

Ho: La satisfacción laboral del empleado con la empresa no está relacionada con la edad del vendedor.

H2: La satisfacción laboral del empleado con la empresa sí está relacionada con la edad del vendedor. 


\section{Resultados}

Tabla 2. Situación media de los empleados respecto a su satisfacción laboral

\begin{tabular}{l|l}
\hline \hline \multicolumn{1}{c|}{ Variables } & Medias \\
\hline Nivel de satisfacción del empleado con la empresa & 7.66 \\
Nivel de satisfacción del empleado con el salario remunerado & 7.25 \\
Nivel de satisfacción del empleado con el entorno de trabajo & 7.11 \\
Nivel de satisfacción del empleado con los compañeros de trabajo & 7.41 \\
Nivel de satisfacción del empleado con su trabajo & 7.28 \\
Nivel de satisfacción del empleado con su jefe & 8.02 \\
Nivel de satisfacción del empleado con sus beneficios. & 7.18 \\
N válido (según lista) & \\
\hline \hline
\end{tabular}

${ }^{1}$ En una escala de 1 = totalmente insatisfecho a $10=$ totalmente satisfecho Fuente: Elaboración propia

La Tabla 2 muestra que las tres principales variables donde los empleados se encuentran satisfechos son, en orden de importancia, Nivel de satisfacción del empleado con su jefe, con una media de 8.02 en una escala de $1=$ total desacuerdo a $10=$ total acuerdo como límites, seguida de la variable Nivel de satisfacción con la empresa con una media de $7.66 \mathrm{y}$, finalmente, Nivel de satisfacción con los compañeros de trabajo con una media de 7.41.

Tabla 3. Nivel de satisfacción del empleado con la empresa y Género del encuestado

\begin{tabular}{|c|c|c|c|c|c|}
\hline & & & \multicolumn{2}{|c|}{ Género del encuestado } & \multirow[b]{2}{*}{ Total } \\
\hline & & & Femenino & Masculino & \\
\hline \multirow[t]{6}{*}{$\begin{array}{l}\text { Nivel de satisfacción del } \\
\text { empleado con la empresa }\end{array}$} & totalmente insatisfecho & $\begin{array}{l}\text { Recuento } \\
\% \text { del total }\end{array}$ & $\begin{array}{l}6 \\
1.5 \%\end{array}$ & $\begin{array}{l}6 \\
1.5 \%\end{array}$ & $\begin{array}{l}12 \\
3.0 \%\end{array}$ \\
\hline & casi siempre insatisfecho & $\begin{array}{l}\text { Recuento } \\
\% \text { del total }\end{array}$ & $\begin{array}{l}44 \\
11.0 \%\end{array}$ & $\begin{array}{l}22 \\
5.5 \%\end{array}$ & $\begin{array}{l}66 \\
16.5 \%\end{array}$ \\
\hline & moderadamente insatisfecho & $\begin{array}{l}\text { Recuento } \\
\% \text { del total }\end{array}$ & $\begin{array}{l}53 \\
13.3 \% \\
\end{array}$ & $\begin{array}{l}42 \\
10.5 \%\end{array}$ & $\begin{array}{l}95 \\
23.8 \%\end{array}$ \\
\hline & medianamente satisfecho & $\begin{array}{l}\text { Recuento } \\
\% \text { del total }\end{array}$ & $\begin{array}{l}65 \\
16.3 \% \\
\end{array}$ & $\begin{array}{l}62 \\
15.5 \% \\
\end{array}$ & $\begin{array}{l}127 \\
31.8 \%\end{array}$ \\
\hline & casi siempre satisfecho & $\begin{array}{l}\text { Recuento } \\
\% \text { del total }\end{array}$ & $\begin{array}{l}36 \\
9.0 \%\end{array}$ & $\begin{array}{l}33 \\
8.3 \%\end{array}$ & $\begin{array}{l}69 \\
17.3 \%\end{array}$ \\
\hline & totalmente satisfecho & $\begin{array}{l}\text { Recuento } \\
\% \text { del total }\end{array}$ & $\begin{array}{l}0 \\
.0 \%\end{array}$ & $\begin{array}{l}30 \\
7.5 \%\end{array}$ & $\begin{array}{l}30 \\
7.5 \%\end{array}$ \\
\hline Total & & $\begin{array}{l}\text { Recuento } \\
\% \text { del total }\end{array}$ & $\begin{array}{l}204 \\
51.1 \%\end{array}$ & $\begin{array}{l}195 \\
48.9 \%\end{array}$ & $\begin{array}{l}399 \\
100.0 \%\end{array}$ \\
\hline
\end{tabular}

${ }^{1}$ En una escala de $1=$ totalmente insatisfecho a $6=$ totalmente satisfecho Fuente: Elaboración propia 
En la Tabla 3 se observa que los hombres alcanzan un mayor porcentaje de satisfacción con respecto a las mujeres, esto en cuanto a la empresa con que laboran. Además un $43.3 \%$ de los encuestados se manifiestan satisfechos en comparación con un $56.6 \%$ que no está totalmente satisfecho con la empresa en la que trabaja.

\section{Chi-cuadrada de independencia}

Queremos establecer si existe una relación entre la satisfacción laboral del empleado con la empresa y la variable género del vendedor.

\section{Formulación de la hipótesis}

Ho: La satisfacción laboral del empleado con la empresa es independiente del sexo del vendedor

H1: La satisfacción laboral del empleado con la empresa es dependiente del sexo del vendedor

El nivel de significancia que se establece es de un alfa de $0.05\left(\mathrm{X}^{2}\right.$ tabla $=21.026)$ en una prueba estadística en que se elige la siguiente fórmula: $\mathrm{X}^{2}=$ (O-E) ${ }^{2} / \mathrm{E}$, y para comparar, se establece que: si $\mathrm{X}^{2}$ (calculada) es menor o igual a 21.06 se acepta la hipótesis nula, y si es mayor a 21.06 se acepta la hipótesis alternativa, razón por la cual se requiere el resultado de la siguiente tabla:

Tabla 3a. Pruebas de chi-cuadrado

\begin{tabular}{|l|l|l|l|}
\hline \multicolumn{1}{l|}{} & \multicolumn{1}{|c|}{ Valor } & \multicolumn{1}{|c|}{ gl } & $\begin{array}{c}\text { Sig. asintótica } \\
\text { (bilateral) }\end{array}$ \\
\hline Chi-cuadrado de Pearson & $38.625^{\mathrm{a}}$ & 5 & .000 \\
Razón de verosimilitudes & 50.339 & 5 & .000 \\
Asociación lineal por lineal & 19.398 & 1 & .000 \\
N de casos válidos & 399 & & \\
\hline
\end{tabular}

a. 0 casillas (.0\%) tienen una frecuencia esperada inferior a 5. La frecuencia mínima esperada es 5.86 .

En vista de que el resultado es 38.625 se concluye que la satisfacción laboral del empleado con la empresa es dependiente del género del vendedor, en un valor comparado con el que puede ocurrir por azar, ya que el valor de $\mathrm{p}$ es menor al 0.05 . en otras palabras, la variable satisfacción es dependiente del sexo del vendedor.

En esta misma tabla, se puede observar el resultado de la razón de verosimilitud con el cual se elaborará una prueba diagnóstica mediante un diseño de casos y controles por medio del algoritmo de Bayes ; éste sería como sigue:

Ho: La satisfacción laboral del empleado con la empresa no está relacionada con el sexo del vendedor. 
H1: La satisfacción laboral del empleado con la empresa sí está relacionada con el sexo del vendedor.

Observándose que la razón de verosimilitud es de 50.339 con un valor de Sig. asintótica (bilateral) de 0.00 que nos dice que es significativo, debe aceptarse la hipótesis alternativa, esto es que la satisfacción laboral del empleado con la empresa sí esá relacionada con el sexo del vendedor, y se puede observar que la razón de verosimilitud es mas conservadora evitando el error que se puede cometer si se hiciera la evaluación solo desde la prueba de chi-cuadrada.

Tabla 4. Nivel de satisfacción del empleado con la empresa y Edad en años ${ }^{1}$

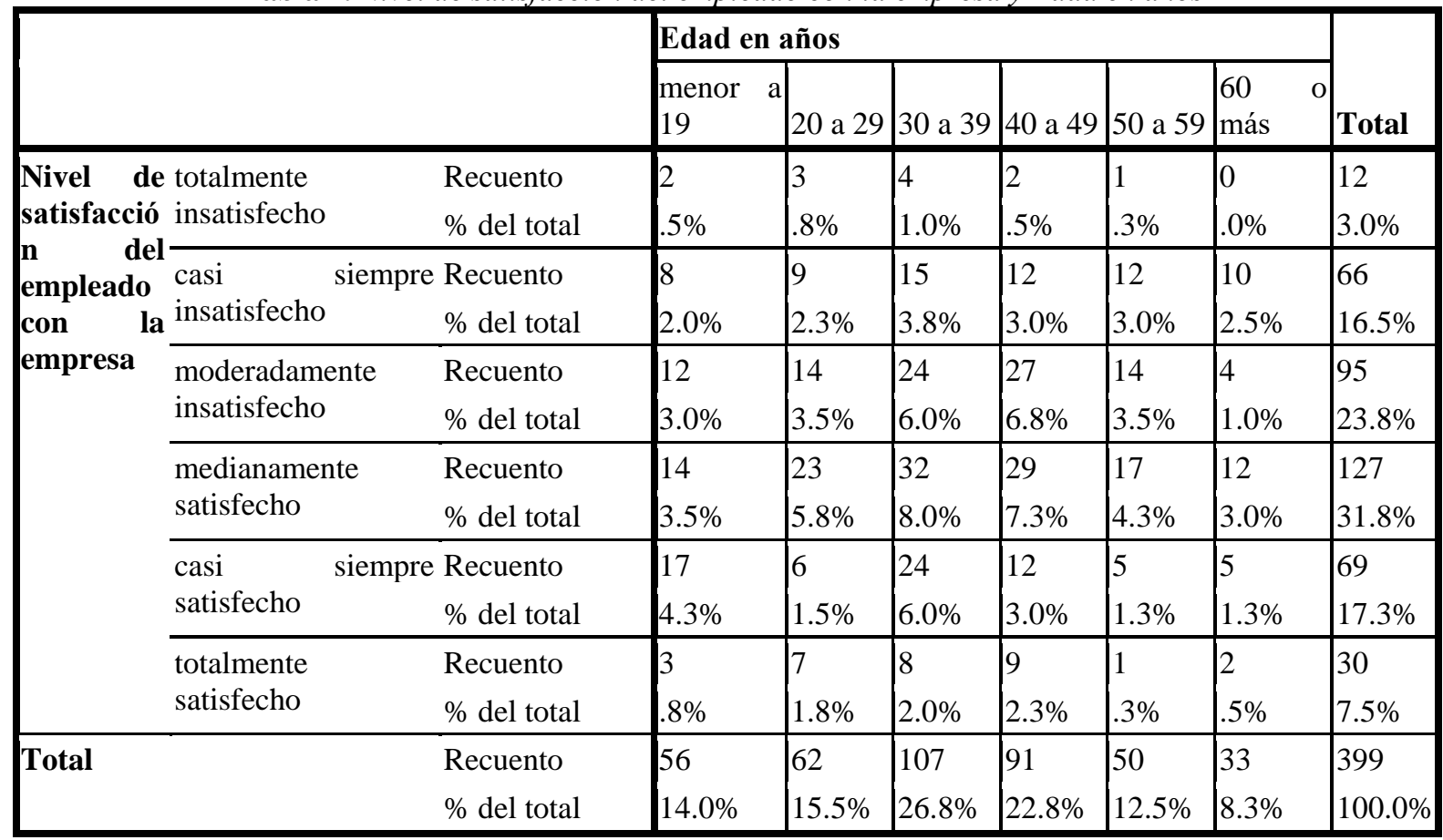

${ }^{1}$ En una escala de $1=$ totalmente insatisfecho a $6=$ totalmente satisfecho Fuente: Elaboración propia

En la Tabla 4, se manifiesta que la mayor satisfacción del empleado con la empresa se experimenta antes de los 50 años de edad y luego disminuye. También se observa que la satisfacción se encuentra en la medianía, en una ambivalencia entre un poco de satisfacción y un poco de insatisfacción, aún y cuando se encuentra ligeramente en el área de satisfecho medianamente.

\section{Chi-cuadrada de independencia}

Queremos establecer si existe una relación entre la satisfacción laboral del empleado con la empresa y la variable edad del vendedor. 


\section{Formulación de la hipótesis}

Ho: La satisfacción laboral del empleado con la empresa es independiente de la edad del vendedor.

H1: La satisfacción laboral del empleado con la empresa es dependiente de la edad del vendedor.

El nivel de significancia que se establece es de un alfa de $0.05\left(\mathrm{X}^{2}\right.$ tabla $=55.758)$ en una prueba estadística en la que se elige la siguiente fórmula:

$\mathrm{X}^{2}=(\mathrm{O}-\mathrm{E})^{2} / \mathrm{E}$ y para comparar, se establece que: si $\mathrm{X}^{2}$ (calculada) es menor o igual a 55.758, se acepta la hipótesis nula, y si es mayor a 55.758 se acepta la hipótesis alternativa, por lo que se requiere el resultado de la siguiente tabla:

Tabla 4a. Pruebas de chi-cuadrado

\begin{tabular}{|l|l|l|l|}
\hline & \multicolumn{1}{|c|}{ Valor } & \multicolumn{1}{|c|}{ Gl } & \multicolumn{1}{|c|}{$\begin{array}{c}\text { Sig. asintótica } \\
\text { (bilateral) }\end{array}$} \\
\hline Chi-cuadrado de Pearson & $30.794^{\mathrm{a}}$ & 25 & .196 \\
Razón de verosimilitudes & 31.432 & 25 & .175 \\
Asociación lineal por lineal & 2.612 & 1 & .106 \\
N de casos válidos & 399 & & \\
\hline
\end{tabular}

a. 10 casillas $(27.8 \%)$ tienen una frecuencia esperada inferior a $5 . \mathrm{La}$ frecuencia mínima esperada es .99 .

Puesto que el resultado es 30.794 , se concluye que la satisfacción laboral del empleado con la empresa es independiente de la edad del vendedor, en un valor comparado con el que puede ocurrir por azar, ya que el valor de Sig. asintótica (bilateral) es mayor al 0.05 , queriendo decir esto que hay una independencia entre las variables.

En esta misma tabla, se puede observar el resultado de la razón de verosimilitud con el cual se elaborará una prueba diagnóstica mediante un diseño de casos y controles por medio del algoritmo de Bayes ; la cual ésta sería como sigue:

Ho: La satisfacción laboral del empleado con la empresa no está relacionada con la edad del vendedor.

H1: La satisfacción laboral del empleado con la empresa si está relacionada con la edad del vendedor.

Se observa que la razón de verosimilitud es de 31.432 con un valor de Sig. asintótica (bilateral) de 0.175 , lo que nos dice que no es significativo. Por esta razón debe aceptarse la hipótesis nula, esto es que la satisfacción laboral del empleado con la empresa no está relacionada con la edad del vendedor. Se puede observar también que la razón de verosimilitud es más conservadora, evitando el error que se puede cometer si se hiciera la evaluación sólo desde la prueba de chi-cuadrada. 
Tabla 5. Nivel de satisfacción del empleado con el salario remunerado y Género del encuestado

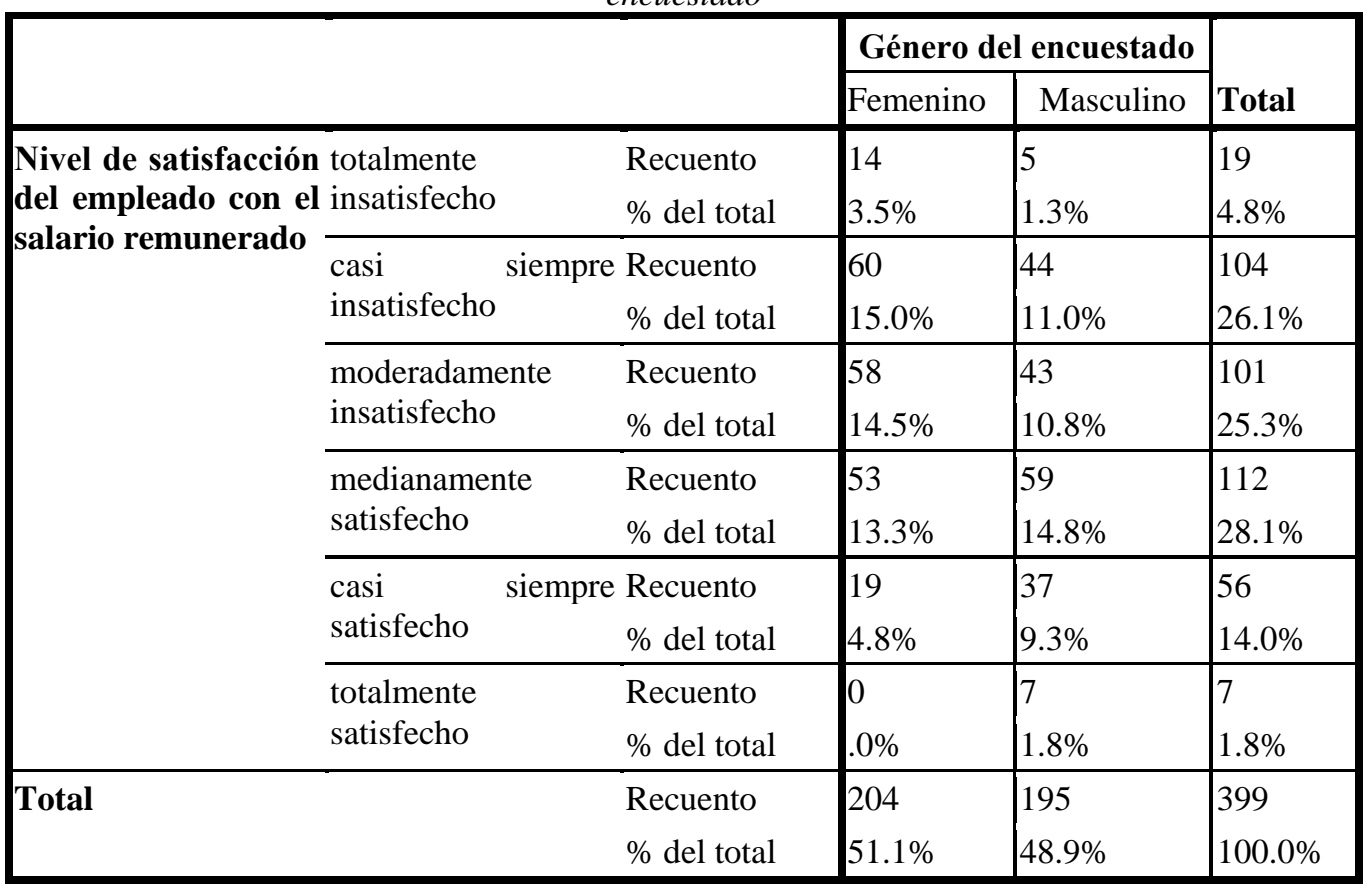

En la Tabla 5 se observa que en cuanto al salario remunerado las mujeres están menos satisfechas con este, aún y cuando se encuentran más insatisfechas que los hombres en general. Aquí es necesario aclarar que durante la encuesta se encontró que los salarios de las mujeres eran inferiores a los de los hombres, lo cual puede ser motivo de otro estudio y sobre todo de un sesgo a tomar en cuenta.

También se observa que hay una gran satisfacción con el salario, sin embargo la opinión de los varones es más dispersa.

\section{Chi-cuadrada de independencia}

Queremos establecer si existe una relación entre la satisfacción laboral del empleado con el salario remunerado y la variable sexo del vendedor.

\section{Formulación de la hipótesis}

Ho: La satisfacción laboral del empleado con el salario remunerado es independiente del sexo del vendedor.

H1: La satisfacción laboral del empleado con el salario remunerado es dependiente del sexo del vendedor.

El nivel de significancia que se establece es de un alfa de $0.05\left(\mathrm{X}^{2}\right.$ tabla $=21.026)$ en una prueba estadística en la que se elige la siguiente fórmula: 
$\mathrm{X}^{2}=(\mathrm{O}-\mathrm{E})^{2} / \mathrm{E}$ y para comparar, se establece que: si $\mathrm{X}^{2}$ (calculada) es menor o igual a 21.06, se acepta la hipótesis nula, y si es mayor a 21.06 se acepta la hipótesis alternativa, por lo que se requiere el resultado de la siguiente tabla:

Tabla 5a. Pruebas de chi-cuadrado

\begin{tabular}{|l|c|c|c|}
\hline & Valor & gl & $\begin{array}{c}\text { Sig. asintótica } \\
\text { (bilateral) }\end{array}$ \\
\hline Chi-cuadrado de Pearson & $21.868^{\mathrm{a}}$ & 5 & .001 \\
Razón de verosimilitudes & 24.859 & 5 & .000 \\
Asociación lineal por lineal & 17.823 & 1 & .000 \\
$\mathrm{~N}^{\circ}$ de casos válidos & 399 & & \\
\hline
\end{tabular}

a. 2 casillas (16.7\%) tienen una frecuencia esperada inferior a $5 . \mathrm{La}$ frecuencia mínima esperada es 3.42 .

En vista de que el resultado es $21.868^{\mathrm{a}}$, se concluye que la satisfacción laboral del empleado con el salario remunerado es dependiente del sexo del vendedor, en un valor comparado con el que puede ocurrir por azar; ya que el valor de Sig. asintótica (bilateral) es menor al 0.05 esto quiere decir que la variable satisfacción es dependiente de la variable sexo.

En esta misma tabla, se puede observar el resultado de la razón de verosimilitud con el cual se elaborará una prueba diagnóstica mediante un diseño de casos y controles por medio del algoritmo de Bayes, la cual sería como sigue:

Ho: La satisfacción laboral del empleado con el salario remunerado no está relacionada con la edad del vendedor.

H1: La satisfacción laboral del empleado con el salario remunerado si está relacionada con la edad del vendedor.

Se observa que la razón de verosimilitud es de 24.859 con un valor de Sig. asintótica (bilateral) de 0.00 , lo que nos dice que es significativo, por lo que debe aceptarse la hipótesis alternativa; esto es que la satisfacción laboral del empleado con el salario remunerado si está relacionada con el sexo del vendedor.Se puede observar que la razón de verosimilitud es más conservadora, evitando el error que se puede cometer si se hiciera la evaluación sólo desde la prueba de chi-cuadrada.

\section{Conclusion}

Los resultados obtenidos al aplicar el cuestionario a 399 profesionales de venta en la Región Sureste del Estado de Coahuila, en México, presentan resultados preliminares, siendo los más relevantes los siguientes: la satisfacción laboral si está relacionada con el género, destacando que los hombres de la muestra están más satisfechos en comparación con las mujeres. Las mujeres perciben un salario menor que los hombres, por lo que se destaca 
su insatisfacción con el empleo. Considerando la variable edad no se encuentran diferencias estadísticamente significativas entre los entrevistados, por lo que se concluye que la satisfacción laboral es independiente de la edad del vendedor.

El propósito de esta investigación fue precisamente el revisar las percepciones de la satisfacción del cliente interno, específicamente de los profesionales de venta, pues existe evidencia en otros entornos, y para los autores del presente estudio era importante contar con evidencia empírica para seguir avanzando en el proyecto.

\section{References:}

1. Carnegie, D. (1996). Cómo Ganar Amigos e Influir sobre las Personas. Mexico: SUDAMERICANA.

2. Hernández, S. R., Fernández, C. C., \& Baptista, L. P. (2014). Metodología de la Investigación (Sexta Edición). México: McGrawHill.

3. Kotler, P., \& Armstrong, G. (2008). Fundamentos de Marketing. México: Pearson. Prentice Hall.

4. Kotler, P., \& Keller, K. (2006). Dirección de Marketing. México: Pearson.

5. Marchand, H. (9 de Octubre de 1998). Horacio Marchand. Recuperado el 13 de Junio de 2013, de http://www.horaciomarchand.com/menuarticulos/1-categoria-estrategia-management/556-articulo19981009 culturainstantanea

6. Schiffman, L. G., \& Lazar Kanuk, L. (2010). Comportamiento del Consumidor (DÉCIMA EDICIÓN ed.). México: PEARSON EDUCACIÓN.

7. Serraf, G. (1988). Diccionario Metodológico de Mercadotencia. México: Trillas.

8. Varoglu \& Eser (2006). "How service Employees can be treated as Internal Customer in Hospitality industry". Businesss review, 30-35.

9. Webb, D. J., Mohr, L. A., \& Harris, K. E. (2008). A re-examination of socially responsible consumption and its measurement. Journal of Business Research, 91-98. 